\title{
Aptamer Based Microfluidic Chip for Microbial Detection
}

\author{
Cong-Xiao ZHANG ${ }^{1, a,{ }^{*}}$, Xue-Fei LV ${ }^{2, b}$, Hong QING ${ }^{2, c}$ and Yu-Lin DENG ${ }^{2, d}$ \\ ${ }^{1}$ School of Pharmacy, Qingdao University, Qingdao, Shandong 266021, China \\ ${ }^{2}$ School of Life Science, Beijing Institute of Technology, Beijing 100081, China \\ azhangcongixao@foxmail.com, ${ }^{b} x$ uefeilv@163.com, chqing@bit.edu.cn, deng@bit.edu.cn
}

Keywords: Aptamer, Microfluidic chip, Microbial detection

\begin{abstract}
Aptamer becomes a novel biosensor for detection of small chemical molecules, biological molecules, and cells. The integrating of aptamer biosensors with microfulidic chip for detection have attracted much attention due to their advantage of miniaturization, sensitivity and high specifity. Microbial detection is very important in the field of clinical diagnosis, environmental analysis and biodefense. It is highly desirable to develop a sensitive and rapid method for microbial detection. In this paper, we develop a novel method using aptamer based microfluidic chip for rapid and specific recognition of Lactobacillus acidophilus. It is will contribute to the microbial detection portably and rapidly.
\end{abstract}

\section{Introduction}

Microbial infection can bring lots of diseases, and cause great economic losses in the public health all over the world [1]. As a result, microbial detection plays an important role in the prevention and treatment for infectious diseases.

The common method utilized in microbial detection is the conventional culture of microorganisms, such as microscopic observation, biochemical reactions and molecular identification. The process contains many steps including microbial culture, separating, purification and identification, leading to consuming time and high costs [2, 3].

In order to overcome the disadvantages in traditional methods, there are urgent needs to develop novel method to detect the microorganism with rapidness and sensitivity. The rapid development of aptamers and microfluidic chips provides a new approach to solve the problem through integrating aptamer biosensors with microfluidic chips.

Aptamers are nucleic acids that can bind to the target with good specifity and affinity, inculding small molecules, biomacromolecules and even whole cells [4]. Aptamers are seleted from a random sequence oligonucleotide library via systematic evolution of ligands by exponential enrichment (SELEX) in vitro [5]. Aptamers are more appealing because of the unique advantages over antibodies, such as synthesis in vitro, nontoxicity, easier modification, and good maintenance [6]. With the feature of high specifity and affinity to the target molecule, aptamers become popular utilized in the field of biosensors as specific recognition elements [7].

Microfluidic chips are able to handle microliter or nanoliter volumes of fluids, using micro-channels with dimensions of micrometers. Microfluidic devices have the advantage of a greater surface-to-volume ratio, a faster mass and energy transfer, and manipulating and controlling the small volume of fluids precisely and flexibly [8]. Microfluidic chips have been widely applied in analytical chemistry, biotechnology, synthetic chemistry, and so on [9]. An ongoing central issue of microfluidics is integrating various function modules into the microfluidic chip, aiming to Lab-On-a-chip in order to realize the multiple function of the common biological and chemical experiment lab [10].

The infectious disease caused by the harmful microorganism can lead to the mortality of human beings and huge economic losses [11]. Developing rapid and sensitive method for microbial detection is helpful to prevent the infectious disease. The purpose of our study in this paper is to develop a 
novel method using aptamer based microfluidic chip for rapid and specific recognition of the typical bacteria stain Lactobacillus acidophilus.

\section{Materials and Methods}

Bacterial Culture. The typical stain Lactobacillus acidophilus was purchased from CGMCC ( China General Microbiological Culture Collection Center ). The bacteria were cultured in typical MRS medium following the CGMCC's instructions under aerobic conditions at the temperature of $37^{\circ} \mathrm{C}$.

The bacteria were cultured in the medium overnight, and the subculture of bacteria was obtained. The cells were then centrifuged at the speed of 2500rpm and at the temperature of $4^{\circ} \mathrm{C}$. After that, the bacteria were washed twice in the buffer ( $5 \mathrm{mM} \mathrm{KCl,} 1 \mathrm{mM} \mathrm{CaCl}_{2}, 1 \mathrm{mM} \mathrm{MgCl}_{2}, 140 \mathrm{mM} \mathrm{NaCl}, 20$ $\mathrm{mM}$ Tris-acetate buffered saline, $\mathrm{pH}$ 7.4). At last, the bacteria were resuspended in the binding buffer.

Bacteria Preparation . The bacterial cells were stained by fluorescein isothiocyanate ( FITC ) solutions at the temperature of $37^{\circ} \mathrm{C}$ for two hours, and washed twice by the buffer ( $20 \mathrm{mM}$ Tris-acetate , $\mathrm{pH} 7.4$ ). FITC was dissolved into acetone and pyridine immediately. And then the bacteria was incubated with the FITC solution for $15 \mathrm{~min}$ in the temperature $37^{\circ} \mathrm{C}$. At last, the bacterial cells were rinsed three times with PBS solutions.

Fabrication of Microfluidic Chip . The UV-curable adhesive NOA81 ( Norland Optical Adhesive ) was studied to fabricate the microfluidic chip. NOA81 was cured by UV light exposure to forming a layer of thin film with a micro-structure. And then multilayer films were bonded together to fabricate the microfluidic chip. Next, avidin $(1 \mathrm{mg} / \mathrm{ml})$ in the Tris-acetate buffer was injected into the microfluidic chip and incubated for one minute and then rinsed with binding buffer three times.

The aptamer labeled by biotin targeted to the bacteria was denatured by heating at $94{ }^{\circ} \mathrm{C}$ for one minute. And then the aptamer solution was put on the ice immediately for 10 minutes, following to incubate them for one minute and rinsed with the binding buffer three times.

Microbial Detection in the Microfluidic Chip. Bacterial cell solutions were injected into the microfluidic chip and incubated for one minute, and then rinsed with the washing buffer ( Binding Buffer with $0.05 \%$ BSA ). The fluorescence images of bacterial cells captured by aptamer immobilized in the microfluidic chip were obtained using an Olympus IX71 inverted fluorescence microscope.

\section{Results and Discussions}

Fabrication of Microfluidic Chip. The adhesive NOA81 was investigated as the material to fabricate the microfluidic chip. The black mask was designed as shown in Fig. 1 for fabricating the microfluidic chip by NOA81 for microbial detection using aptamer. The microfluidic chip has four micro-channels consisting four spots in which aptamers were immobilized on the glass surface in order to capture the microorganism specifically.

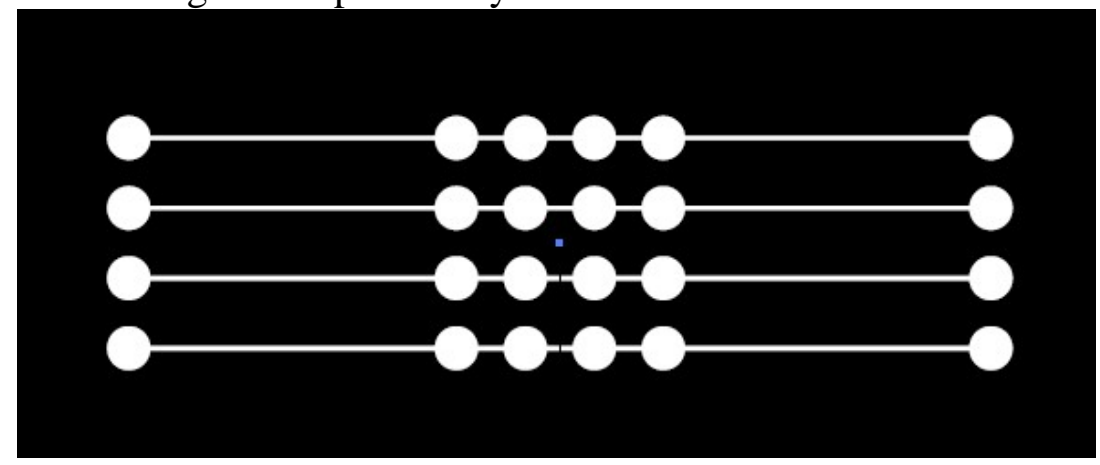

Fig. 1 Design of black mask and photograph of microfluidic chip 
The diameter of detection spot was $500 \mu \mathrm{m}$, and the array spots were used to display the fluorescence signal of the bacteria captured by the aptamer. The width of micro-channel in the microfluidic chip is $50 \mu \mathrm{m}$. The aptamer targeted to the bacteria was modified with a poly $\mathrm{T}$ linker in order to keep the specific conformation of the aptamer targeted to the bacterial cell when it was immobilized on the glass surface of the microfluidic chip. The aptamer based microfluidic chip was fabricated as shown in Fig. 2.

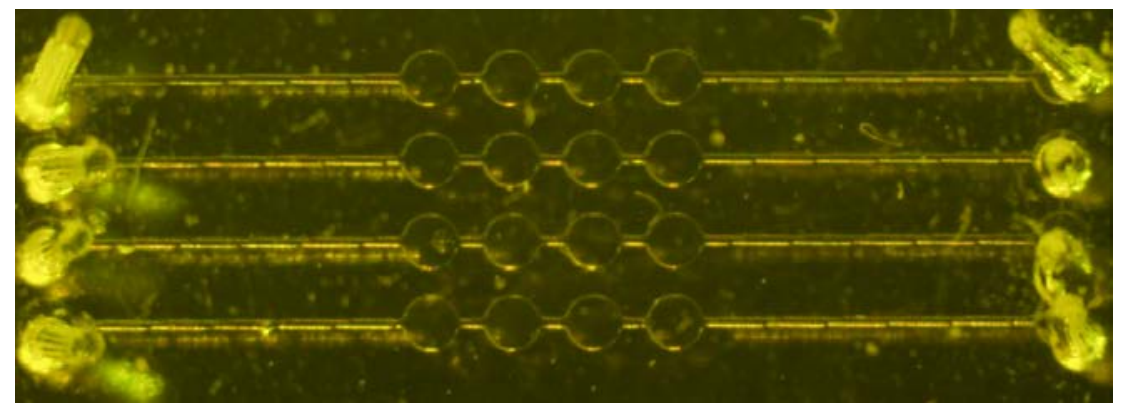

Fig. 2 Photograph of aptamer based microfluidic chip

Microbial Detection in the Microfluidic Chip. The solution of bacteria labeled the FITC was injected into the microfluidic chip. After washing three times, the non-target bacteria will be rinsed off, and Lactobacillus acidophilus will be captured by the aptamer immobilized in the microfluidic chips due to the specific affinity between the aptamer and the bacterial cells.

The fluorescence microscope was utilized to obtain the fluorescence signal of the bacteria captured by the aptamer in the microfluidic chip. As is shown in Fig. 3, the green fluorescent signal was obtained from the horizon of the fluorescent microscope in the microfluidic chip. And the fluorescent signals were distributed evenly in the array spots. It is inferred that the aptamer based microfluidic chip was able to detect the bacteria Lactobacillus acidophilus rapidly.

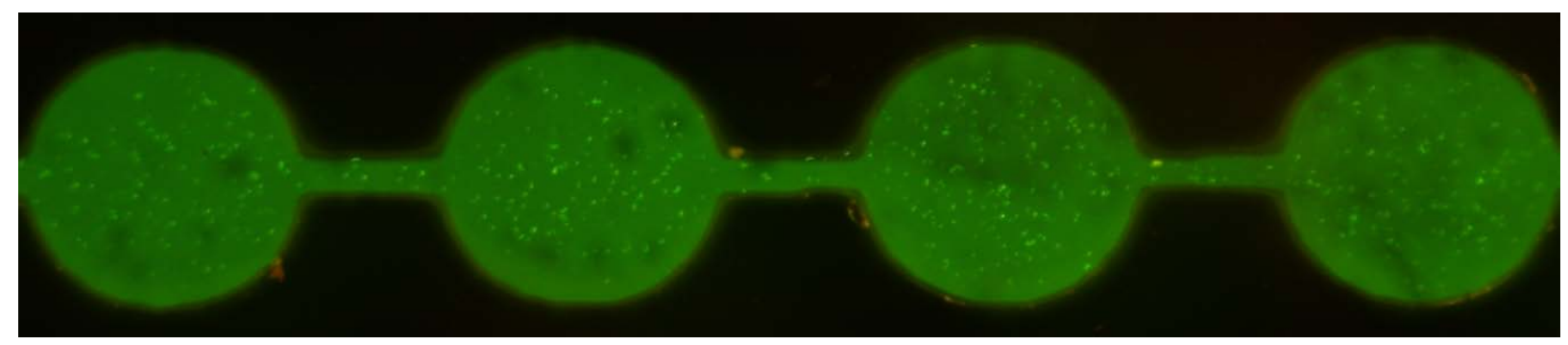

Fig. 3 Fluorescence image of L.achidophilus captured by aptamer in the microfluidic chip (20×)

\section{Conclusions}

In this paper, the aptamer was used as the specific recognition element integrated into the microfluidic chip for microbial detection. The results showed that the aptamer based on microfluidic chip was able to detect Lactobacillus acidophilus by the specific affinity between aptamer and bacterial cells. It is helpful to develop the potable, rapid, and sensitive device for microbial detection.

\section{Acknowledgements}

This work was financially supported by the National Natural Science Foundation of China (21275019) and Postdoctoral Applied Research Programme Funded by Qingdao Government (2015151). 


\section{References}

[1] O. Lazcka, F. J. D. Campo and F. X. Munoz, Pathogen detection: A perspective of traditional methods and biosensors, Biosensors and Bioelectronics. 22 (2007) 1205-1217.

[2] G. A. Zelada-Guill n, J. Riu, A. D zg n and F. X. Rius, Immediate Detection of Living Bacteria at Ultralow Concentrations Using a Carbon Nanotube Based Potentiometric Aptasensor, Angewandte Chemie International Edition. 48 (2009) 7334-7337.

[3] Y. X. Wang, Z. Z. Ye, C. Y. Si and Y. B. Ying, Application of Aptamer Based Biosensors for Detection of Pathogenic Microorganisms, Chinese J. Anal. Chem. 40 (2012) 634-642.

[4] T. Ku, T. Zhang, H. Luo, T. M. Yen, P. Chen, Y. Han and Y. Lo, Nucleic Acid Aptamers: An Emerging Tool for Biotechnology and Biomedical Sensing, Sensors-Basel. 15 (2015) 16281-16313.

[5] J. J. Wu, Y. Y. Zhu, F. Xue, Z. L. Mei, L. Yao, X. Wang, L. Zheng, J. Liu, G. D. Liu, C. F. Peng and W. Chen, Recent trends in SELEX technique and its application to food safety monitoring, Microchim. Acta. 181 (2014) 479-491.

[6] F. Radom, P. M. Jurek, M. P. Mazurek, J. Otlewski and F. Jelen, Aptamers: Molecules of great potential, Biotechnol. Adv. 31 (2013) 1260-1274.

[7] M. Famulok and G. Mayer, Aptamer Modules as Sensors and Detectors, Accounts Chem. Res. 44 (2011) 1349-1358.

[8] P. N. Nge, C. I. Rogers and A. T. Woolley, Advances in microfluidic materials, functions, integration, and applications, Chem. Rev. 113 (2013) 2550-2583.

[9] M. D. Leonida and I. Kumar, Microfluidics: from Engineering to Life Sciences, Curr. Nanosci. 8 (2012) 458-473.

[10] D. Mark, S. Haeberle, G. Roth, F. von Stetten and R. Zengerle, Microfluidic lab-on-a-chip platforms: requirements, characteristics and applications, Chem. Soc. Rev. 39 (2010) 1153-1182.

[11] E. Torres-Chavolla and E. C. Alocilja, Aptasensors for detection of microbial and viral pathogens, Biosensors and Bioelectronics. 24 (2009) 3175-3182. 